\title{
Distinctive characteristics of his bundle potentials in patients with atrioventricular nodal reentrant tachycardia
}

\author{
Fu Guan ${ }^{1,2}$, Ardan M. Saguner ${ }^{1}$, Daniel Hofer ${ }^{1}$, Thomas Wolber ${ }^{1}$, \\ Alexander Breitenstein ${ }^{1}$, Nazmi Krasniqi ${ }^{1,3}$, Urs Eriksson ${ }^{1,3}$, \\ Jan Steffel ${ }^{1}$, Corinna Brunckhorst ${ }^{1}$, Firat Duru ${ }^{1,4^{4}}$ \\ ${ }^{1}$ Arrhythmia and Electrophysiology Division, Department of Cardiology, \\ University Heart Center, Zurich, Switzerland \\ ${ }^{2}$ Department of Cardiology, Beijing Shijitan Hospital, Capital Medical University, Beijing, China \\ ${ }^{3}$ Department of Cardiology, GZO Regional Health Center Wetzikon, Switzerland \\ ${ }^{4}$ Center for Integrative Human Physiology, University of Zurich, Switzerland
}

\begin{abstract}
Background: His bundle $(H B)$ potentials vary in amplitude and duration in patients with and without slow pathways. The aim of this study was to determine the characteristics of HB potentials and to elucidate whether they can provide clues for identification of slow pathway (SP).

Methods: The present research prospectively studied the electrophysiological findings of 162 patients with symptomatic atrioventricular nodal reentrant tachycardia (AVNRT) due to slow-fast or fast-slow type and atrioventricular reentrant tachycardia (AVRT). Maximal HB potential (HBmax, HB with the highest amplitude) among HB cloud was recorded in both groups. For AVNRT patients, the following were measured: (1) AH interval at the "jump" during programmed atrial stimulation (A2H2, taken as a reflection of SP conduction time); (2) Distance from HBmax to the successful SP ablation site (HBmax-ABL) and from HBmax to the ostium of coronary sinus (HBmax-CSO).

Results: HBmax was $0.29 \pm 0.10 \mathrm{mV}$ in AVNRT patients, whereas it was $0.17 \pm 0.05 \mathrm{mV}$ in AVRT group ( $p$ < 0.0001). Likewise, the HBmax duration was $22 \pm 5 \mathrm{~ms}$ in AVNRT group and $16 \pm 3 \mathrm{~ms}$ in AVRT group $(p<0.0001)$. The area under the receiver operating characteristic curve of HBmax amplitude in AVNRT patients was 0.86 and the optimal HBmax cut-off to predict AVNRT was $\geq 0.22 \mathrm{mV}$ with a sensitivity of 0.78 and specificity of 0.84 . HBmax-CSO was positively correlated with $H B m a x-A B L$, and HBmax-ABL was positively correlated with $A 2 H 2$.

Conclusions: HBmax amplitudes were higher and durations longer in patients with AVNRT, as compared to those with AVRT. Moreover, the distance between HBmax and successful ablation site was positively correlated with the SP conduction time and with the distance from HBmax to the CSO. (Cardiol J) Key words: tachycardia, atrioventricular nodal reentry, slow pathway, His bundle, catheter ablation
\end{abstract}

Address for correspondence: Prof. Dr. Firat Duru, Arrhythmia and Electrophysiology Division, Department of Cardiology, University Heart Center Zurich, Raemistrasse 100, CH-8091, Zurich, Switzerland, tel: +41 442553565 , e-mail: firat.duru@usz.ch

Received: 4.05.2021 Accepted: 27.08.2021 Early publication date: 23.09.2021

This article is available in open access under Creative Common Attribution-Non-Commercial-No Derivatives 4.0 International (CC BY-NC-ND 4.0) license, allowing to download articles and share them with others as long as they credit the authors and the publisher, but without permission to change them in any way or use them commercially. 


\section{Introduction}

Since its introduction in 1992, catheter ablation of the slow pathway (SP) has been considered the first-line treatment in symptomatic patients with atrioventricular (AV) nodal reentrant tachycardia (AVNRT) [1]. The procedure is usually performed using fluoroscopy but three-dimensional (3D) mapping can provide further help by identifying the anatomical structures that are relevant for the ablation [2]. In some patients with AVNRT it is also possible to record SP potentials in the perinodal region [3]. High-resolution mapping of the Triangle of Koch may increase the overall safety and efficacy of the ablation procedure [4].

The possibility to record His bundle (HB) potentials in humans was reported more than 5 decades ago [5]. In patients with AV conduction disturbances, the site of the block can be determined by recording the time delay between the $\mathrm{HB}$ signal and the earliest ventricular activation on surface electrocardiogram. The amplitude and duration of the HB signal depend on multiple factors, such as the type of catheter used, interelectrode spacing and orientation of the catheter tip. Despite widespread use of HB potentials in daily electrophysiology practice, detailed assessments of their signal characteristics in AVNRT patients are lacking. The aim of this study is to determine the distinctive features of the HB potentials, and elucidate if they provide clues for identification of patients with SP, an electrical prerequisite for the development of AVNRT.

\section{Methods}

\section{Study patients}

Electrophysiological findings of consecutive patients with symptomatic AVNRT due to slow-fast type or fast-slow type reentry and AV reentrant tachycardia (AVRT) due to an accessory pathway were studied between December 2018 to September 2020. The study data was prospectively collected in the Catheter Ablation Registry at the University Heart Center Zurich, which was approved by the Institutional Ethical Committee.

\section{Electrophysiological study findings}

All electrophysiological studies were performed in the fasting, non-sedated state. After local anesthesia, multielectrode catheters were introduced percutaneously through the right femoral vein and positioned in high right atrial, right ventricular and $\mathrm{HB}$ positions, followed by measurements of basic electrophysiological parameters. AV nodal effective refractory period (AVNERP) was measured at progressively shortening intervals following 8 drive trains at $600 \mathrm{~ms}$. An atrio-His (AH) jump, suggestive of the presence of an SP, was identified, if there was a $50 \mathrm{~ms}$ or more increase in $\mathrm{H} 1 \mathrm{H} 2$ interval by using $10 \mathrm{~ms}$ decrements of A1A2. The longest conduction time of the fast pathway (A1H1) was measured as the last premature extrastimulus coupling interval before an $\mathrm{AH}$ jump. The shortest conduction time of the slow pathway (A2H2) was defined as the first premature extrastimulus coupling interval after an $\mathrm{AH}$ jump. All measurements were performed under baseline conditions without administration of isoprenaline.

If a tachycardia was not inducible at baseline, isoproterenol was administered. Various stimulation techniques were used in both groups to ensure the induction and reproducibility of the clinical tachycardia. V-A-V response during ventricular entrainment was used to exclude an atrial tachycardia.

\section{Mapping and catheter ablation}

After diagnosis of AVNRT or AVRT, a 7-Fr quadripolar, steerable radiofrequency ablation catheter (Navistar $4 \mathrm{~mm}$, Biosense Webster, Irvine, CA, USA) was introduced into the right atrium. Prior to mapping, calibration for respiratory compensation was performed. The filter settings for bipolar signals were set at the $16-500 \mathrm{~Hz}$ range. Using the ablation catheter, anatomical locations showing $\mathrm{HB}$ potentials were extensively searched and tagged point-by-point using the CARTO 3D electroanatomical mapping system (Biosense Webster, Irvine, CA, USA) to determine the His cloud (Fig. 1). For each patient, there was a minimum of 8 points that determined the His cloud. The HB signal with the maximal amplitude was labeled as HBmax. Every point was double-checked by offline analysis to confirm correct annotation. 3D mapping hallmarks of HBmax, the central point of the coronary sinus ostium (CSO), and the site of SP ablation (ABL) in the standard right anterior oblique view (RAO $30^{\circ}$ ) were annotated for distance identification. The amplitude and duration of HBmax between the two groups were measured using endocardial tracings at a recording speed of $400 \mathrm{~mm} / \mathrm{s}$.

For SP ablation, the inferior/posterior aspect of the interatrial septum adjacent to the CSO was targeted. The local signal typically showed a small, low frequency atrial and a sizeable, high-frequency ventricular deflection, and rarely an SP potential, but no HB signal, as described by previous publications [6-8]. The ablation site was selected if there was adequate safety distance from the lowest $\mathrm{HB}$ 


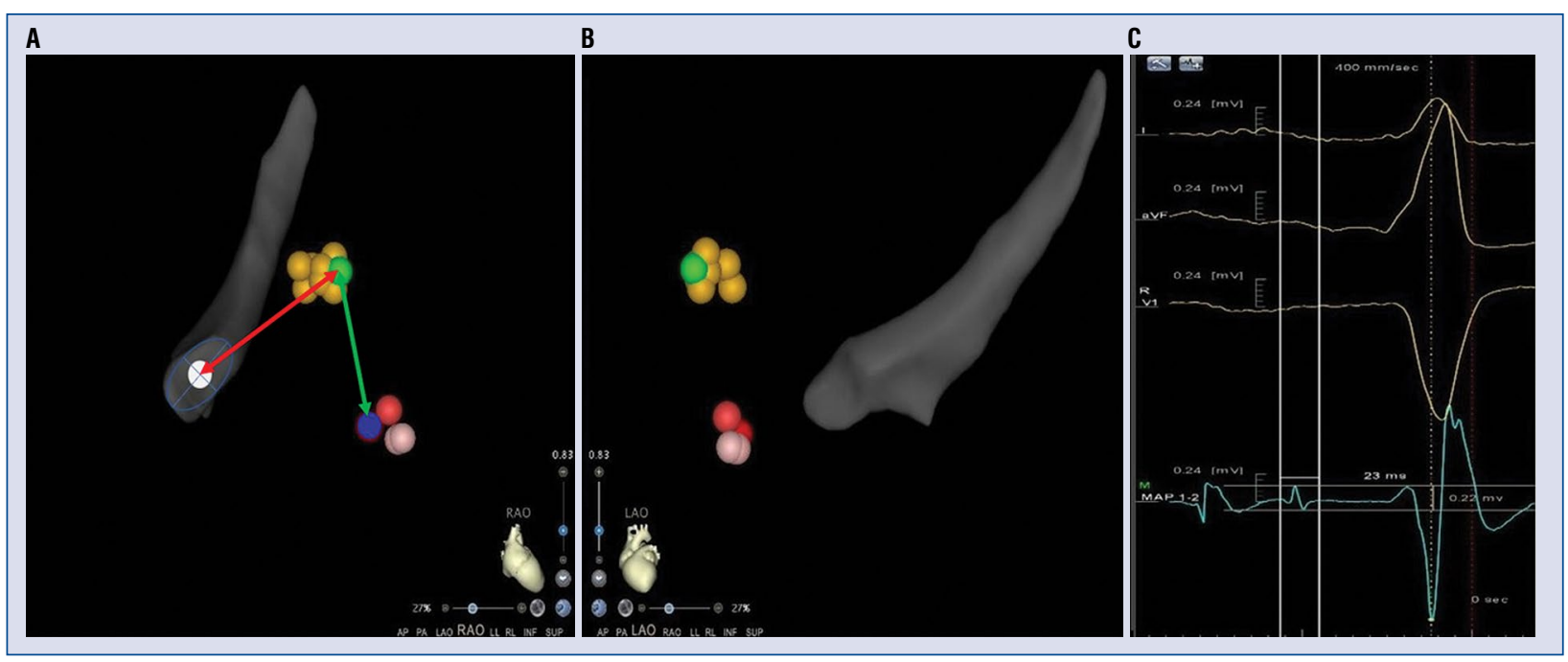

Figure 1. Anatomical hallmarks (His cloud, coronary sinus and ablation points) are shown on a CARTO 3D electroanatomical map in (A) right anterior oblique (RAO, 30 ) and (B) left anterior oblique (LAO, 60 $0^{\circ}$ views and endocardial electrograms with a recording speed of $400 \mathrm{~mm} / \mathrm{s}$ are shown in (C). HB - bundle of His; HBmax - maximal His potential; CSO - ostium of coronary sinus; ABL — ablation site. The maximal HB potential (HBmax) was marked as a green point within the His cloud (multiple yellow points). The best site with induction of slow junctional beats among various ablation points (in red) was labeled as $A B L$ (blue point). The center of CSO was also marked (white point). The red arrow line indicates the distance between HBmax and CSO. The green arrow line indicates the distance between HBmax and the slow pathway ablation site. Due to CSO variability, its center was determined as the intersection of the longest diameter and the shortest diameter.

point. The site of SP ablation (ABL, blue point in 3D map) with best response showing slow junctional beats during ablation was identified for each patient. AVRT patients were ablated along either the right or left $\mathrm{AV}$ valvular annulus, depending on the location of the accessory pathway.

Radiofrequency energy was applied with an overall period of $60-120 \mathrm{~s}$ on the ablation site with a power setting in the range of $30-50 \mathrm{~W}$ for both groups. The endpoint for AVNRT ablation was noninducibility of the tachycardia. For patients with an AH jump (with or without an echo beat) after ablation, the SP ablation sites were often multiple. Since this study also aims at the SP ablation site, patients who had a residual SP were excluded from analysis. For AVRT patients, abolishing antegrade and/or retrograde accessory pathway conduction was considered as the endpoint of the procedure. Electrophysiologic study was repeated at least 20 min after last ablation to confirm the success of the procedure. In order to prevent thromboembolic complications, intravenous heparin was administered during the procedure and subcutaneous unfractionated heparin was given post-operatively during bed rest. Patients who underwent ablation of left-sided accessory pathways were prescribed acetylsalicylic acid for 4-6 weeks. All patients were discharged from the clinic either on the same day of the procedure or on the day after. They were followed up at our cardiac arrhythmia clinic or by the referring physicians after discharge.

\section{Statistical analysis}

Data were expressed as means \pm standard deviation. For continuous variables, data were expressed as median (interquartile range), and Kruskal-Wallis test was performed to examine the difference among groups. Mann-Whitney U test was used to test the difference in continuous variables between groups. The correlations between measurements were examined by linear regression analysis. For the HBmax measurements that significantly differed between AVNRT and AVRT groups, receiver operating characteristic (ROC) curve analysis was used to compare the predicted accuracy and determine cut-off values with optimal performance. Statistical significance was set at $\mathrm{p}<0.005$.

\section{Results}

\section{Patient characteristics}

A total of 162 patients were finally enrolled in the study. Of these, 112 (57 \pm 22 years; 45 males $)$ 
Table 1. Electrophysiologic parameters in AVNRT and AVRT patients.

\begin{tabular}{|c|c|c|c|c|c|}
\hline \multirow[t]{2}{*}{ Parameters } & \multicolumn{2}{|c|}{ AVNRT } & \multicolumn{2}{|c|}{ AVRT } & \multirow[t]{2}{*}{$\mathbf{P}$} \\
\hline & Prior ablation & Post ablation & Prior ablation & Post ablation & \\
\hline $\mathrm{N}$ & \multicolumn{2}{|c|}{$\mathrm{N}=112,45$ male } & \multicolumn{2}{|c|}{$\mathrm{N}=50,29$ male } & $<0.005^{*}$ \\
\hline Age [years] & \multicolumn{2}{|c|}{$57 \pm 22$} & \multicolumn{2}{|c|}{$33 \pm 12$} & $>0.005^{*}$ \\
\hline \multirow[t]{3}{*}{ AVBCL [ms] } & $369 \pm 75$ & $398 \pm 45$ & $363 \pm 74$ & $366 \pm 70$ & $>0.005^{*}$ \\
\hline & & & & & $<0.005^{1 \#}$ \\
\hline & & & & & $>0.005^{2 \#}$ \\
\hline \multirow[t]{3}{*}{ VABCL [ms] } & $374 \pm 56$ & $380 \pm 51$ & $365 \pm 19$ & $367 \pm 43$ & $>0.005^{*}$ \\
\hline & & & & & $<0.005^{1 \#}$ \\
\hline & & & & & $>0.005^{2 \#}$ \\
\hline $\mathrm{A} 1 \mathrm{H} 1$ interval $[\mathrm{ms}]$ & $159 \pm 38$ & NA & NA & NA & \\
\hline $\mathrm{A} 2 \mathrm{H} 2$ interval [ms] & $250 \pm 48(n=107)^{@}$ & NA & NA & NA & \\
\hline \multirow[t]{2}{*}{ HV interval [ms] } & $45 \pm 6$ & $45 \pm 5$ & NA & $43 \pm 4$ & $<0.005^{*}$ \\
\hline & & & & & $<0.005^{\#}$ \\
\hline Tachycardia CL [ms] & $388 \pm 58$ & NA & $372 \pm 65$ & NA & $<0.005^{*}$ \\
\hline
\end{tabular}

Data were presented as median (lower to upper quartile; range) or mean \pm standard deviation. AVNRT — atrioventricular nodal reentrant tachycardia; AVRT — atrioventricular reentrant tachycardia; AVBCL — atrioventricular block cycle length; VABCL — ventriculoatrial block cycle length; $\mathrm{A} 1 \mathrm{H} 1$ - fast pathway conduction time; $\mathrm{A} 2 \mathrm{H} 2$ - slow pathway conduction time; $\mathrm{HV}$ - conduction time from His Bundle to earliest ventricular activation on surface electrocardiogram; $\mathrm{CL}$ - cycle length; $\mathrm{NA}$ - no relevant measurement available; *Compared between AVNRT and AVRT groups prior or post-ablation; \#Compared within group prior or post-ablation; 1\#In AVNRT group; 2\#In AVRT group; @Note that the Pearson correlation analysis related to A2H2 was performed in 107 typical AVNRT patients, excluding 5 patients fast-slow type AVNRT who had no observed antegrade slow pathway conduction

had AVNRT and the remaining 50 had AVRT $(33 \pm 12$ years; 29 males $)$. No patients were enrolled in the latter group if they had evidence for the presence of concomitant dual AV nodal physiology. Eight patients had coronary artery disease (6 in AVNRT group). Four patients had sick sinus syndrome with an implanted pacemaker, one had dilated cardiomyopathy and one had valvular heart disease, all of whom in the AVNRT group. In the remaining 121 patients (74 AVNRT, 47 AVRT), there was no evidence of an underlying structural heart disease. All patients had normal systolic left ventricular function (left ventricular ejection fraction [LVEF] $>50 \%$ ), except for one AVNRT patient with cardiomyopathy having an LVEF of $36 \%$. All antiarrhythmic drugs were discontinued prior to electrophysiological study latest on the previous day prior to the procedure. In the AVNRT group, 5 fast-slow type AVNRTs were identified and 107 slow-fast AVNRTs were diagnosed prior to ablation. In the AVRT group, 12 Wolff-Parkinson-White syndrome patients were found to have pre-excited electrocardiogram at baseline. For concealed accessory pathways, 16 patients demonstrated anterograde concealment, 16 demonstrated retrograde concealment, and 6 demonstrated both anterograde and retrograde concealment. Regarding the localization of the accessory pathways around the mitral and tricuspid annuli, there were 6 accessory pathways in the right anterior septum, 4 in the right mid-septum, 9 in the right posterior septum, 10 in the right sided free wall (4 right posterior or right posterolateral, 3 right lateral, 3 right anterior), 4 in the left posterior septum, 1 in the left mid-septum, 16 in the left lateral free wall (13 left lateral or left anterolateral, 3 left posterior or left posterolateral).

The catheter ablation procedure was acutely successful and uneventful in all patients in both AVNRT and AVRT cohorts. Twelve-lead surface electrocardiography parameters and electrophysiological measurements prior to and after ablation in patients of both groups were shown in Table 1.

\section{HB potential characteristics in AVNRT patients compared with AVRT patients}

There were significant differences in HBmax amplitude and duration between the AVNRT (slow-fast and fast-slow type) and AVRT groups after adjusting for age and gender, as shown in Figure 2. The HBmax amplitude was $0.29 \pm 0.10 \mathrm{mV}$ in AVNRT patients, whereas it was $0.17 \pm 0.05 \mathrm{mV}$ in the AVRT group $(\mathrm{p}<0.0001)$. Likewise, the HBmax duration was $22 \pm 5 \mathrm{~ms}$ in the AVNRT group 


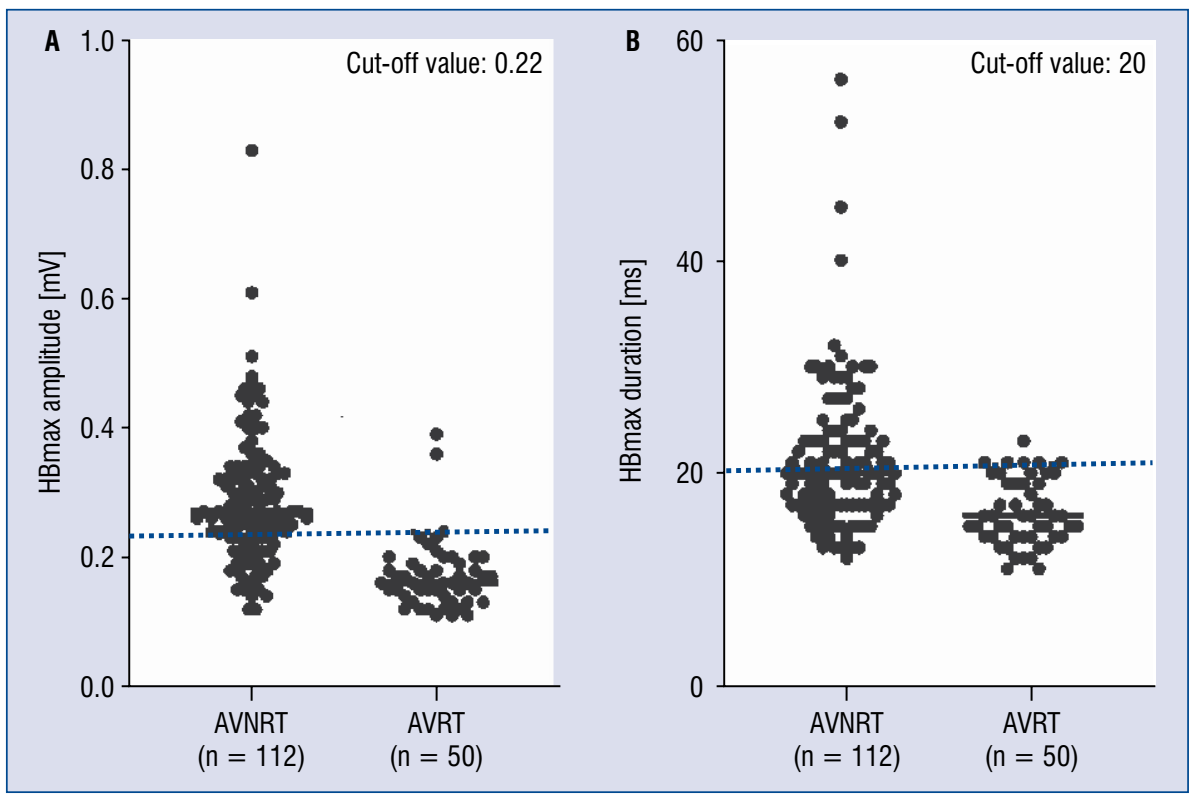

Figure 2. Scatterplot of cut-off values of maximal His potential (HBmax) amplitude (A) and HBmax duration (B) in AVNRT patients; AVNRT — atrioventricular nodal reentrant tachycardia; AVRT — atrioventricular reentrant tachycardia.
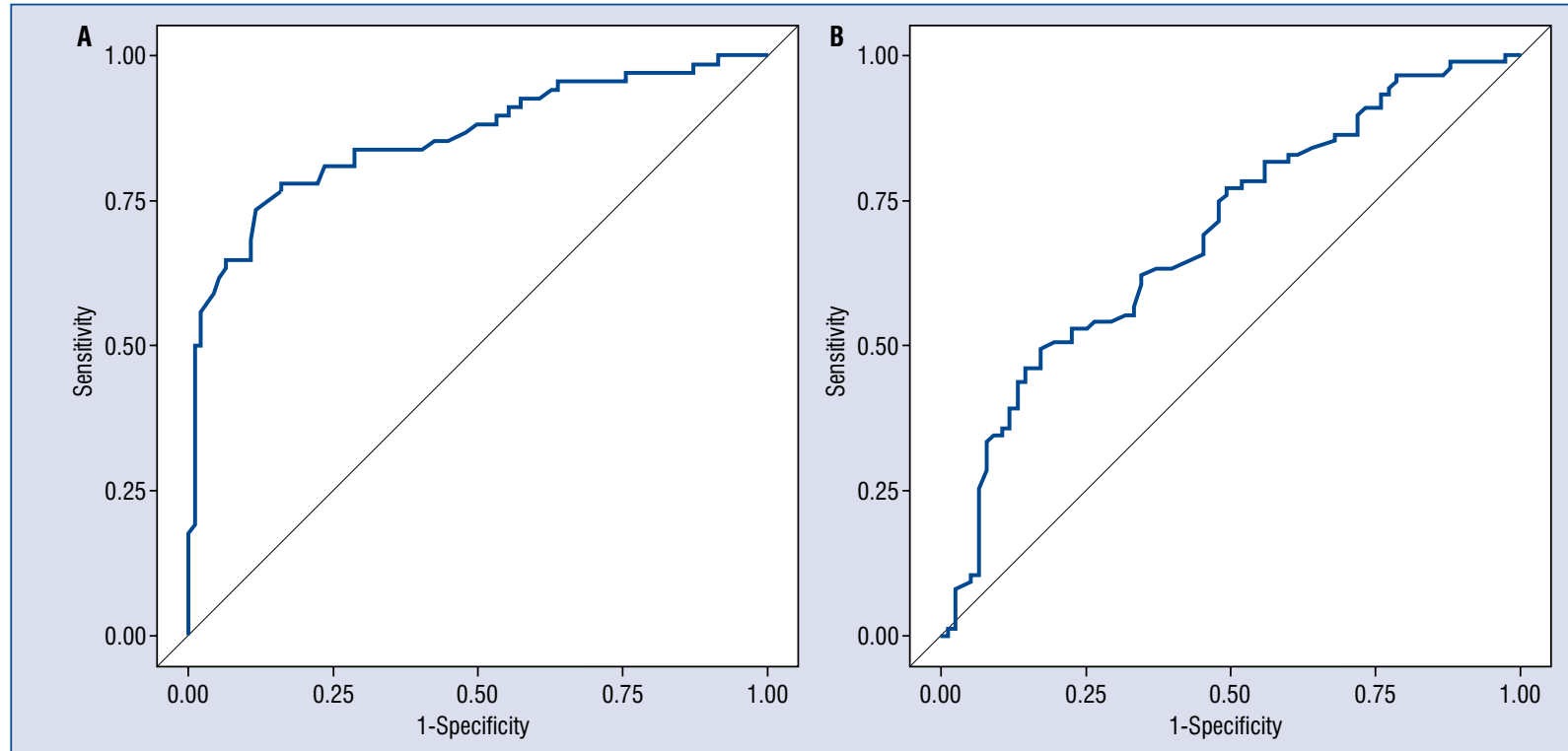

Figure 3. Receiver operating characteristic curve analysis for the optimal cut-off values of maximal His potential (HBmax) amplitude (A) and HBmax duration (B).

and $16 \pm 3 \mathrm{~ms}$ in the AVRT group ( $<<0.0001$ ) Moreover, in five AVNRT patients with fast-slow type reentry, HBmax amplitude was $0.36 \pm 0.09 \mathrm{mV}$ and duration was $24 \pm 2.8 \mathrm{~ms}$, which were significantly different than those values in AVRT patients $(\mathrm{p}<0.0001)$.

It was then sought to define the diagnostic value of HBmax amplitude and duration for dis- crimination of AVNRT due to SP from AVRT. After adjusting for age and gender, linear logistic regression analysis revealed that HBmax amplitude and duration were independent predictors for AVNRT (Fig. 3). The area under the ROC curve (AUC) of HBmax amplitude in AVNRT patients was 0.86, and the optimal cut-off to predict AVNRT was equal to or greater than $0.22 \mathrm{mV}$ with a sensitivity of 0.78 


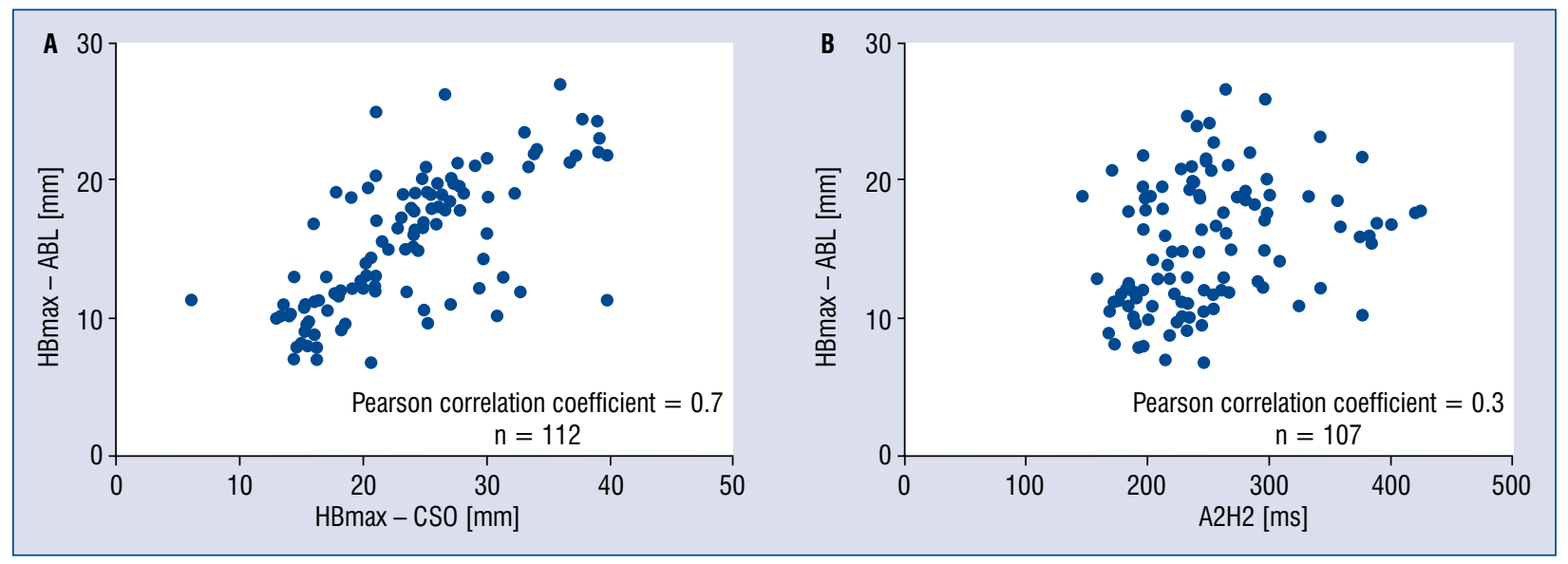

Figure 4. Pearson correlations between the distance maximal His potential (HBmax) site from the successful ablation site and coronary sinus ostium (CSO) (A), and the distance HBmax site from the successful ablation site and slow pathway conduction (A2H2) (B); $\mathrm{ABL}$ - ablation site.

and specificity of 0.84 (Fig. 2). Meanwhile, the AUC of HBmax duration was 0.69 , and the optimal cutoff to predict AVNRT was equal to or greater than $20 \mathrm{~ms}$ with a sensitivity of 0.49 and specificity of 0.83 .

\section{Electrophysiological and anatomical correlations of $\mathrm{HBmax}$ in AVNRT patients}

Three-dimensional CARTO maps showed $15.4 \pm 4.9 \mathrm{~mm}$ distance between the successful ablation site and the HBmax recording site. The distance between the center of the CSO and the $\mathrm{HBmax}$ recordings site was $23.5 \pm 5.6 \mathrm{~mm}$. The mean A2H2 interval with an SP conduction was $250 \pm 48 \mathrm{~ms}$. The distance between HBmax and CSO was positively correlated with the distance between HBmax and the successful ablation site $\left(\mathrm{r}^{2}=0.7174,95 \%\right.$ confidence interval $[\mathrm{CI}] 0.6101$ to $0.7989, \mathrm{p}=0.0004)$, in accordance with the previous publication (Fig. 4) [9]. The HBmax distance from the successful ablation site was positively correlated with the shortest SP conduction time (A2H2) $\left(\mathrm{r}^{2}=0.3368,95 \%\right.$ CI 0.1515 to 0.4993 , $\mathrm{p}<0.00001)$, but it was not correlated with HBmax amplitude or duration. The distance between HBmax and CS ostium was not correlated with A2H2. Likewise, it was neither correlated with HBmax amplitude nor HBmax duration.

\section{Ablation procedure and follow up}

Ablation was successfully performed in all patients enrolled. No significant cardiac adverse effects occurred during energy application, and all had preserved AV conduction. The overall duration of each study was 1-3 hours. No patient had tachy- cardia recurrence or apparent electrocardiography parameter changes with a mean post-ablation period of 6 months.

\section{Discussion}

\section{Main findings}

The present study had several findings: First, it was demonstrated that HBmax amplitudes were higher and HBmax durations were longer in patients with AVNRT, as compared to those measurements in patients with AVRT. These measurements might be helpful for primary evaluation of the two arrhythmic conditions with a relatively high sensitivity and specificity. Second, the distance between HBmax and successful ablation site was positively correlated with the SP conduction time and with the distance from HBmax to the CSO. These findings may be helpful in choosing the optimal SP site for ablation.

The higher HBmax amplitudes and longer HBmax durations were not only observed in patients with typical AVNRT but were also observed in patients with atypical AVNRT due to fast-slow type reentry, as compared to those measured in patients with AVRT. On the other hand, these measurements were not significantly different in patients with left-sided, right-sided, or septal accessory pathways. The differential diagnosis of atypical AVNRT from AVRT could be challenging since extra electrophysiologic maneuvers were often required, such as parahisian pacing and ventricular entrainment $[10,11]$. The HBmax measurements might provide primary clues for 


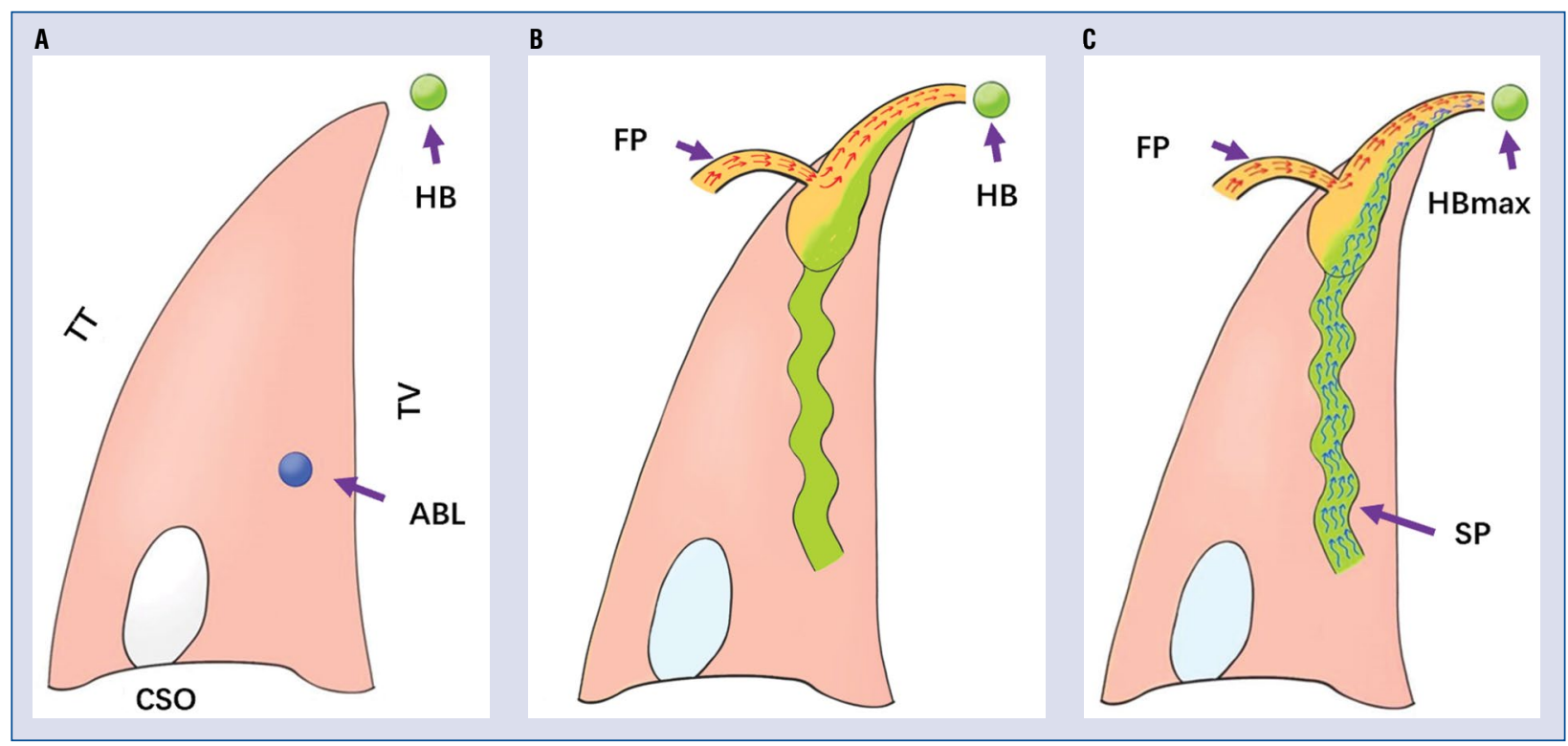

Figure 5. Schematic illustration of dual pathway anterograde conduction. The schematic represents the Koch's triangle and adjacent area; $\mathbf{A}$. Triangle of Koch in the right anterior oblique projection and the location of $\mathrm{HBmax}$ and $\mathrm{ABL}$ hallmarks; B. Fast pathway conduction of sinus activity to distal HB in patients without dual atrioventricular conduction; C. The dual pathway conduction of sinus activity to distal HB through longitudinal dissociation in patients with atrioventricular nodal reentrant tachycardia. The red arrows represent fast pathway conduction, and the blue curved arrows represent slow pathway conduction. The yellow area represents $\mathrm{Cx} 43(-)$ cells, and the green area the $\mathrm{C} \times 43(+)$ cells; TT — tendon of Todaro; TV — tricuspid valve; CSO — ostium of coronary sinus; ABL — successful ablation site; HB - His bundle; HBmax — maximal His bundle recording site; FP — fast pathway; SP — slow pathway.

dual AVN physiology and lead to corresponding electrophysiological study protocol priority for mechanism identification.

\section{Distinctive HB potential in AVNRT patients}

It has been known for more than 30 years that longitudinal dissociation within the posterior $\mathrm{AV}$ nodal region is the main mechanism, which gives rise to localized reentry and AVNRT [12]. Despite this, the signal characteristics of the HB potentials in human beings with an SP have not been scrutinized in depth to date. Only previous observations from rabbit AVNRT models show an augmentation of the HB signal amplitude during pacing with short premature coupling intervals [13]. The current study, using electroanatomical 3D mapping of the $\mathrm{AV}$ nodal and $\mathrm{HB}$ region, fills the knowledge gap for patients with AVNRT and is in line with previous observations from animal models. In the present study, particular attention was paid to obtaining a complete representation of the HB cloud by taking 8 or more annotation points along the area of HB that showed distinct HB potentials. The observed augmentation of the HBmax signal (especially its amplitude) in patients with AVNRT possibly reflects an electrical region that represents the continuation of two distinct electrical pathways joining together into an enhanced HB deflection. Clearly, this also necessitates proper orientation of the fibers at the bipolar recording site of the His catheter. This illustration is shown in the form of a schematic diagram in Figure 5.

The presence of an SP may also be manifested in some occasions during sinus rhythm. Intermittent occurrence of prolonged PR intervals, which may arise following ectopic beats, may suggest an SP [14]. Ablative elimination of the SP in such patients will restore physiological AV conduction over the fast pathway. Moreover, dual AV nodal non-re-entrant tachycardia, which is characterized by double antegrade conduction from a single sinus nodal activity simultaneously via the slow and fast AV nodal pathways into the ventricle, has been described $[15,16]$. Furthermore, SP potentials prior to the HB deflection can also be detected by electrophysiological catheter during sinus rhythm on some occasions [3].

Meanwhile, immunobiological study on human $\mathrm{AV}$ junction demonstrates differential $\mathrm{Cx} 43(+)$ myocyte distribution, with rightward nodal extensions and HB much more than leftward nodal extensions and compact node (Fig. 5) [17]. These observations provide further proof of the unique 
conduction properties of different parts of AV junctional area, which contribute to longitudinal dissociation and reentrant arrhythmias.

\section{HBmax electrophysiology correlates with related anatomy in AVNRT patients}

The positive correlation between SP conduction time and the distance from HBmax to successful ablation site, as observed in our study, may simply imply a longer conduction time for the SP wavefront to perpetuate due to a longer anatomical distance. This finding is in line with the findings of Dagres et al. [18] who demonstrated that SP with longer conduction intervals was more often ablated in a more inferior area of the Koch's triangle.

The observed difference in tachycardia cycle length between AVNRT and AVRT in the current study is in line with previous observations in adult patients [19]. In contrast, tachycardia cycle length of the two arrhythmic conditions does not differ in pediatric patients [20]. This discrepancy is explained as the conduction of both fast and slow pathways is faster in children than in adults. The adult patients in the study herein, did not manifest a correlation between age and SP conduction time or the distance from HBmax to CSO.

\section{Limitations of the study}

The present study has some limitations. HBmax in this study was observed during sinus rhythm before any electrophysiological maneuvers or medication induction was introduced. This could only give us a preliminary assessment of possible differential diagnosis of dual AVN physiology or AVNRT. Whereas the fundamental evaluation and step-by-step electrophysiological study can never be underestimated. Moreover, since the focus herein was on the effective ablation site compared to other anatomical structures within the triangle of Koch, patients with multiple SPs were excluded. Therefore, we could not comment on whether observations would hold true in patients with slow-slow type reentry.

\section{Conclusions}

HBmax amplitudes are higher and HBmax durations are longer in patients with AVNRT, as compared to those in AVRT. These features may provide preliminary clues to differentiate the two arrhythmic conditions with relatively high sensitivity and specificity. Moreover, the distance between HBmax and successful ablation site is positively correlated with the SP conduction time and with the distance from HBmax to the CSO. These findings are helpful in determining the presence of an $\mathrm{SP}$ and in optimizing SP ablation.

\section{Acknowledgments}

We thank Daria Vdovenko (University of $\mathrm{Zu}$ rich) and Kevin He (University of Michigan) for their constructive advice on statistical analysis.

\section{Conflict of interest: None declared}

\section{References}

1. Jackman WM, Beckman KJ, McClelland JH, et al. Treatment of supraventricular tachycardia due to atrioventricular nodal reentry by radiofrequency catheter ablation of slow-pathway conduction. N Engl J Med. 1992; 327(5): 313-318, doi: 10.1056/ NEJM199207303270504, indexed in Pubmed: 1620170.

2. Del Carpio Munoz F, Buescher TL, Asirvatham SJ. Three-dimensional mapping of cardiac arrhythmias: what do the colors really mean? Circ Arrhythm Electrophysiol. 2010; 3(6): e6-e11, doi: 10.1161/CIRCEP.110.960161, indexed in Pubmed: 21156773.

3. Haissaguerre M, Gaita F, Fischer B, et al. Elimination of atrioventricular nodal reentrant tachycardia using discrete slow potentials to guide application of radiofrequency energy. Circulation. 1992; 85(6): 2162-2175, doi: 10.1161/01.cir.85.6.2162, indexed in Pubmed: 1591833.

4. Chua K, Upadhyay GA, Lee E, et al. High-resolution mapping of the triangle of Koch: Spatial heterogeneity of fast pathway atrionodal connections. Heart Rhythm. 2018; 15(3): 421-429, doi: 10.1016/j.hrthm.2017.10.030, indexed in Pubmed: 29081398.

5. Damato AN, Lau SH, Berkowitz WD, et al. Recording of specialized conducting fibers (A-V nodal, His bundle, and right bundle branch) in man using an electrode catheter technic. Circulation. 1969; 39(4): 435-447, doi: 10.1161/01.cir.39.4.435, indexed in Pubmed: 5778244.

6. Kay GN, Epstein AE, Dailey SM, et al. Selective radiofrequency ablation of the slow pathway for the treatment of atrioventricular nodal reentrant tachycardia. Evidence for involvement of perinodal myocardium within the reentrant circuit. Circulation. 1992; 85(5): 1675-1688, doi: 10.1161/01.cir.85.5.1675, indexed in Pubmed: 1572026.

7. Jazayeri MR, Hempe SL, Sra JS, et al. Selective transcatheter ablation of the fast and slow pathways using radiofrequency energy in patients with atrioventricular nodal reentrant tachycardia. Circulation. 1992; 85(4): 1318-1328, doi: 10.1161/01.cir.85.4.1318, indexed in Pubmed: 1555276.

8. Wu D, Yeh SJ, Wang CC, et al. A simple technique for selective radiofrequency ablation of the slow pathway in atrioventricular node reentrant tachycardia. J Am Coll Cardiol. 1993; 21(7): 1612-1621, doi: 10.1016/0735-1097(93)90376-c, indexed in Pubmed: 8496527.

9. Geller JC, Biblo LA, Carlson MD. Relation between the AH interval and the ablation site in patients with atrioventricular nodal reentrant tachycardia. Pacing Clin Electrophysiol. 2004; 27(10): 1347-1354, doi: 10.1111/j.1540-8159.2004.00638.x, indexed in Pubmed: 15511243. 
10. Hirao K, Otomo K, Wang X, et al. Para-Hisian pacing. A new method for differentiating retrograde conduction over an accessory AV pathway from conduction over the AV node. Circulation. 1996; 94(5): 1027-1035, doi: 10.1161/01.cir.94.5.1027, indexed in Pubmed: 8790042.

11. Obeyesekere M, Gula LJ, Modi S, et al. Tachycardia induction with ventricular extrastimuli differentiates atypical atrioventricular nodal reentrant tachycardia from orthodromic reciprocating tachycardia. Heart Rhythm. 2012; 9(3): 335-341, doi: 10.1016/j. hrthm.2011.10.015, indexed in Pubmed: 22001824.

12. Patterson E, Scherlag BJ. Longitudinal dissociation within the posterior AV nodal input of the rabbit: a substrate for AV nodal reentry. Circulation. 1999; 99(1): 143-155, doi: 10.1161/01. cir.99.1.143, indexed in Pubmed: 9884391.

13. Zhang Y, Bharati S, Mowrey KA, et al. His electrogram alternans reveal dual-wavefront inputs into and longitudinal dissociation within the bundle of His. Circulation. 2001; 104(7): 832-838, doi: 10.1161/hc3301.092804, indexed in Pubmed: 11502711.

14. Özkartal T, Stehli J, Duru F. Intermittent $\mathrm{PQ}$ prolongation between two premature ventricular complexes: what is the mechanism? Eur Heart J. 2016; 37(32): 2560, doi: 10.1093/eurheartj/ehv733, indexed in Pubmed: 26865477.

15. Natale A, Greenfield RA, Geiger MJ, et al. Safety of slow pathway ablation in patients with long PR interval: further evidence of fast and slow pathway interaction. Pacing Clin Electrophysiol. 1997;
20(6): 1698-1703, doi: 10.1111/j.1540-8159.1997.tb03542.x, indexed in Pubmed: 9227770.

16. Lee SH, Chen SA, Tai CT, et al. Atrioventricular node reentrant tachycardia in patients with a prolonged $\mathrm{AH}$ interval during sinus rhythm: clinical features, electrophysiologic characteristics and results of radiofrequency ablation. J Interv Card Electrophysiol. 1997; 1(4): 305-310, doi: 10.1023/a:1009785127119, indexed in Pubmed: 9869985.

17. Hucker WJ, McCain ML, Laughner JI, et al. Connexin 43 expression delineates two discrete pathways in the human atrioventricular junction. Anat Rec (Hoboken). 2008; 291(2): 204-215, doi: 10.1002/ar.20631, indexed in Pubmed: 18085635.

18. Dagres N, Manolis AS, Maounis T, et al. Site of successful slow pathway ablation relates to clinical tachycardia rate in patients with atrioventricular nodal re-entrant tachycardia. Heart. 2006; 92(1): 115-116, doi: 10.1136/hrt.2004.054056, indexed in Pubmed: 16365362.

19. Wu D, Denes P, Amat-y-Leon F, et al. Clinical, electrocardiographic and electrophysiologic observations in patients with paroxysmal supraventricular tachycardia. Am J Cardiol. 1978; 41(6): 1045-1051, doi: 10.1016/0002-9149(78)90856-1, indexed in Pubmed: 665509.

20. Mills MF, Motonaga KS, Trela A, et al. Is there a difference in tachycardia cycle length during SVT in children with AVRT and AVNRT? Pacing Clin Electrophysiol. 2016; 39(11): 1206-1212, doi: 10.1111/pace.12950, indexed in Pubmed: 27653639. 